Case Report

\title{
Pancreas as Delayed Site of Metastasis from Papillary Thyroid Carcinoma
}

\author{
Mutahir A. Tunio, ${ }^{1}$ Mushabbab AlAsiri, ${ }^{1}$ Khalid Riaz, ${ }^{1}$ and Wafa AlShakweer ${ }^{2}$ \\ ${ }^{1}$ Radiation Oncology, Comprehensive Cancer Center, King Fahad Medical City, Riyadh 59046, Saudi Arabia \\ ${ }^{2}$ Histopathology, Comprehensive Cancer Center, King Fahad Medical City, Riyadh 59046, Saudi Arabia
}

Correspondence should be addressed to Mutahir A. Tunio; mkhairuddin@kfmc.med.sa

Received 4 February 2013; Accepted 4 March 2013

Academic Editors: P. Abraham and S. Nomura

Copyright (C) 2013 Mutahir A. Tunio et al. This is an open access article distributed under the Creative Commons Attribution License, which permits unrestricted use, distribution, and reproduction in any medium, provided the original work is properly cited.

\begin{abstract}
Introduction. Follicular variant (FV) papillary thyroid carcinoma (PTC) has aggressive biologic behavior as compared to classic variant $(\mathrm{CV})$ of PTC and frequently metastasizes to the lungs and bones. However, metastasis to the pancreas is extremely rare manifestation of FV-PTC. To date, only 9 cases of PTC have been reported in the literature. Pancreatic metastases from PTC usually remain asymptomatic or manifest as repeated abdominal aches. Associated obstructive jaundice is rare. Prognosis is variable with reported median survival from 16 to 46 months. Case Presentation. Herein we present a 67-year-old Saudi woman, who developed pancreatic metastases seven years after total thyroidectomy and neck dissection followed by radioactive iodine ablation (RAI) for FV-PTC. Metastasectomy was performed by pancreaticoduodenectomy followed by sorafenib as genetic testing revealed a BRAF V600E mutation. She survived 32 months after the pancreatic metastasis diagnosis. Conclusion. Pancreatic metastases are rare manifestation of FV-PTC and are usually sign of extensive disease and conventional diagnostic tools may remain to reach the diagnosis.
\end{abstract}

\section{Introduction}

Thyroid cancer is the commonest endocrine malignancy, presenting with 23500 new cases per year in the United States and European Union, respectively $[1,2]$. Differentiated thyroid carcinoma (DTC) is the most frequently diagnosed cancer among women in the Middle East, behind only breast cancer, and accounting for more than $10 \%$ of all cancers among women in Saudi Arabia [3].

Papillary thyroid carcinoma (PTC) is the most frequent form of DTC and the follicular variant of PTC (FV-PTC) is more aggressive than the classic variant of PTC. It differs from classical PTC in being follicular growth pattern, higher prevalence of tumor encapsulation, angiovascular invasion, and poorly differentiated areas and a lower rate of lymph node metastases [4].

Pancreas is an extremely rare site of metastasis of PTC. To date, only 9 cases of pancreatic metastasis secondary to PTC have been reported in the literature.
Herein we present a 67-year-old Saudi woman, who developed pancreatic metastasis seven years after total thyroidectomy and neck dissection followed by sorafenib for FVPTC.

\section{Case Presentation}

In November 2009, a 67-year-old Saudi woman presented in our clinic for her routine visit with the complaints of abdominal pain and indigestion. She had noticed these complaints for 2 months and these have been occurring frequently over 2 weeks, for which she was taking antispasmodics and nonsteroidal anti-inflammatory drugs (NSAIDs), but with minimal improvement. Her previous medical history revealed hypertension and diabetes since last 20 years which were controlled on medications. She had no history of smoking and her weight was stable. Her past surgical history showed that she underwent total thyroidectomy and lymph node dissection for follicular variant papillary thyroid carcinoma 


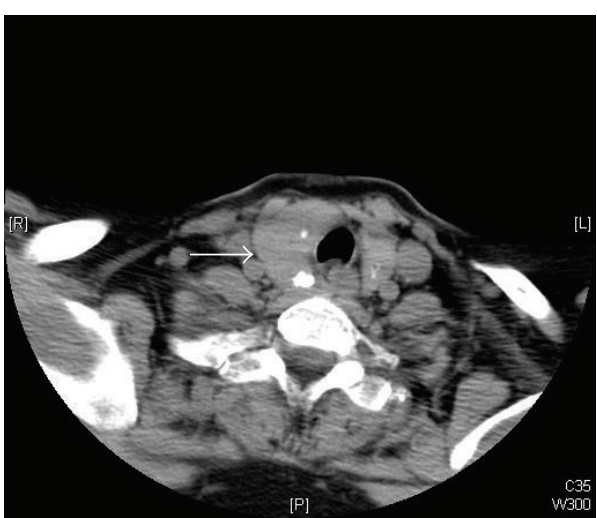

(a)

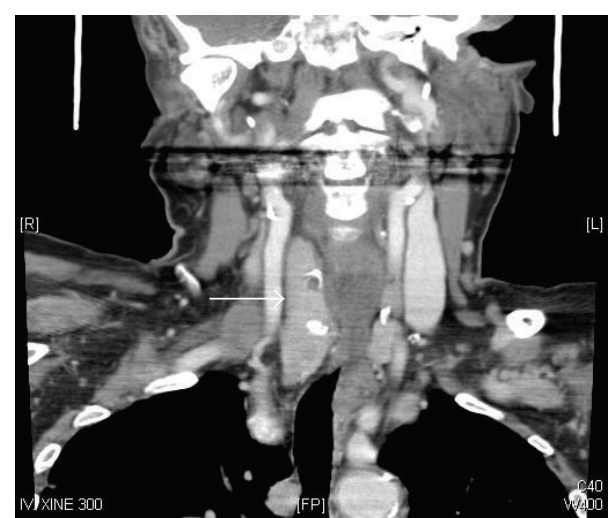

(b)

Figure 1: Computed tomography of neck showing diffuse enlargement of right lobe of thyroid, came out as papillary follicular cell variant thyroid carcinoma pT2N1.

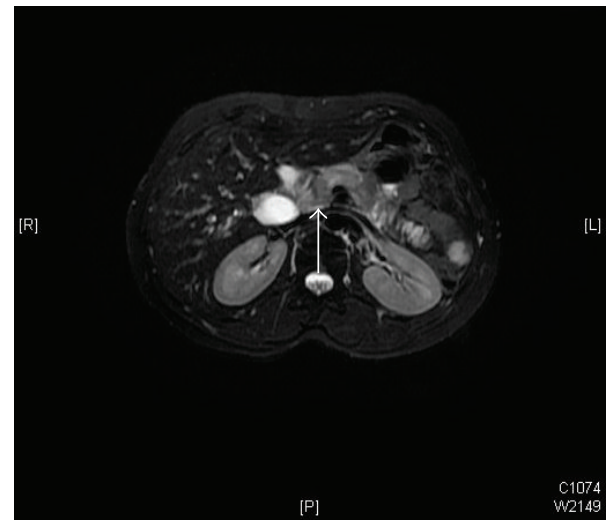

FIGURE 2: Magnetic Resonance Cholangiopancreatography (MRCP) showing a small hypovascular $1.8 \times 1.5 \mathrm{~cm}$ mass in the pancreatic neck, invading the superior mesenteric vein.

pT2N1cM0 (Figure 1) in March 2002, followed by radioactive iodine (RAI) ablation $150 \mathrm{mCi}$ in May 2002, and RAI ablation $150 \mathrm{mCi}$ in July 2003 for raised serum thyroglobulin levels (39.3 ng/mL).

On physical examination, she was in good general condition and her vitals were stable. Per abdomen examination revealed deep tenderness in epigastrium; however, there was no palpable mass or visceromegaly. There was no other palpable cervical lymphadenopathy and examination of chest, heart, nervous system, and pelvic was normal. Clinical differential diagnosis was chronic pancreatitis, primary pancreatic adenocarcinoma, or metastasis. Her hematological, renal, and liver function tests, tuberculin, serum electrolytes, and thyroid stimulating hormone (TSH) and thyroxin (T4) were found within normal limits. Serum tumor markers, carcinoembryonic antigen (CEA), and cancer antigen 19-9 were also normal; however, serum TG levels were raised, that is, $672.8 \mathrm{ng} / \mathrm{mL}$ (normal: $5-25 \mathrm{ng} / \mathrm{mL}$ ). Computed tomography (CT) of abdomen showed decreased enhancement of the neck, body, and tail of the pancreas; while enhancement of the pancreatic head appeared normal and there were bilateral multiple pulmonary metastases. Whole body iodine scintigraphy was noniodine avid. Magnetic Resonance Cholangiopancreatography $(\mathrm{MRCP})$ revealed a small hypovascular lesion in the pancreatic neck measuring $1.8 \times 1.5 \mathrm{~cm}$. It showed low signal intensity in both $\mathrm{T} 1$ and $\mathrm{T} 2$ with no enhancement in the arterial phase and faint enhancement in delayed sequences. Mass was abutting the superior mesenteric vein (SMV) and caused narrowing to its caliber below the confluence with splenic vein; however, superior mesenteric artery (SMA) was found normal in caliber with no sign of invasion (Figure 2). CT guided fine needle aspiration cytology of the mass was performed and it showed papillary nests of eosinophilic tumor cells with intranuclear inclusions. The patient underwent pancreaticoduodenectomy. Histopathology showed $1.6 \times 1.4 \mathrm{~cm}$ metastatic deposit in the neck of the pancreas and immunohistochemistry examination showed the positivity for TG and thyroid transcription factor-1 (TTF1) and made confirmed diagnosis of pancreatic metastasis consistent with FV-PTC (Figure 3). Genetic testing revealed a BRAF V600E mutation. After surgical resection, she was started on sorafenib $400 \mathrm{mg}$ twice daily, which she tolerated well. At time of submission of case report the patient was alive at 36 months postoperatively and was doing fine with partial response in lungs, without recurrence in neck and serum TG levels $39.8 \mathrm{ng} / \mathrm{mL}$.

\section{Discussion}

Pancreas is rare site metastasis. Common malignancies which metastasize to pancreas are renal cell carcinoma, lung, medullary carcinoma of the thyroid, lymphomas, alveolar rhabdomyosarcoma, and esophagus [11]. Metastases to the pancreas from papillary thyroid carcinoma are extremely rare. To date, only 9 cases have been reported in the literature with initial diagnosis of PTC $[5-10,12]$ in Table 1 . Exact pathogenesis is not well known; however, hematogenous route is well supported.

Pancreatic metastases remain asymptomatic for long period before diagnosis or manifest as symptoms like chronic pancreatitis as seen in our patient. At time of presentation, 


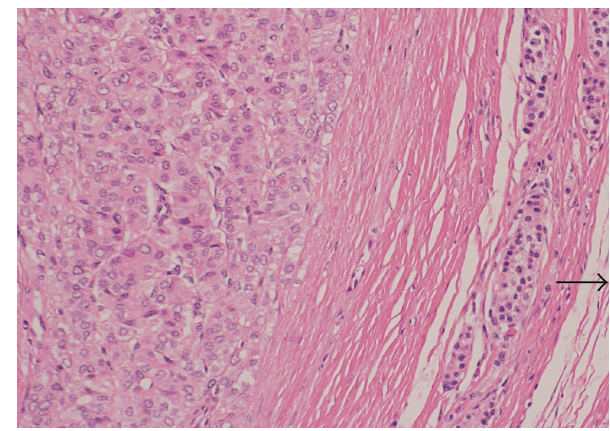

(a)

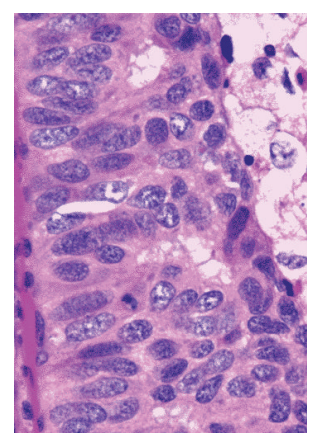

(b)

FIGURE 3: (a) Infiltrating clusters of papillary tumor cells in pancreatic tissue parenchyma $(H \& E \times 100)$ and $(b)$ follicular pattern with abundant cytoplasm $(\mathrm{H} \& \mathrm{E} \times 200)$.

TABLE 1: Cases of pancreatic metastasis secondary to papillary thyroid carcinoma reported from 1991 to 2012.

\begin{tabular}{|c|c|c|c|c|c|}
\hline Author & Age & From time of initial treatment & Variant & Treatment & $\begin{array}{l}\text { Survival after diagnosis } \\
\text { of pancreatic metastasis }\end{array}$ \\
\hline Zhu et al. [4] & NA & NA & Tall cell & Surgical resection & NA \\
\hline Sugimura et al. [5] & 53 years & 7 years after TT + RAI & Classical & Surgical resection & NA \\
\hline Jobran et al. [6] & 53 years male & NA & Tall cell & Surgical resection & NA \\
\hline Angeles-Angeles et al. [7] & 72 years male & NA & Classical & Surgical resection & NA \\
\hline Borschitz et al. [8] & $\begin{array}{c}34 \text { years female } \\
46 \text { years male }\end{array}$ & $\begin{array}{l}9 \text { years after TT + RAI } \\
2 \text { years after TT }+ \text { RAI }\end{array}$ & $\begin{array}{l}\text { Classical } \\
\text { Follicular }\end{array}$ & Surgical resection & 42 months \\
\hline Chen and Brainard [9] & 82 years male & 5 years after $\mathrm{TT}+\mathrm{RAI}$ & Classical & Surgical resection & NA \\
\hline Alzahrani et al. [10] & 56 years male & 6 years after $\mathrm{TT}+\mathrm{RAI}$ & Classical & Sorafenib & 20 months \\
\hline Present case & 67 years & 7 years after TT + RAI & Tall cell & Surgical resection and RAI & Alive at 32 months \\
\hline
\end{tabular}

TT: total thyroidectomy, RAI: radioactive iodine therapy, NA: not mentioned.

pancreatic metastases are usually sign of extensive disease seen in our patient. Conventional diagnostic tools (CT, WBS) may remain fail to reach the diagnosis. However, positron emission tomography-computed tomography (PET-CT) and endoscopic ultrasound (EUS) have shown the sensitivity or detecting pancreatic lesions up to $94-100 \%$, though not performed in our case due to unavailability in institute [13].

Surgical treatment in the form of pylorus sparing pancreaticoduodenectomy relieves the symptoms and may increase the disease free survival (DFS) for isolated pancreatic metastasis. However, adjunct and multikinase inhibitors (sorafenib or sunitinib) may slow the disease progression especially in patients with BRAF V600E mutation in tumor cell lines [14].

In conclusion, pancreatic metastases are rare in FV-PTC and they remain asymptomatic or are associated with nonspecific complaints. Improved diagnostic tools (MRCP, PETCT, and EUS) and immunohistochemistry can be helpful for prompt diagnosis and treatment by means of pancreaticoduodenectomy followed by sorafenib which is associated with increased DFS.

\section{Consent}

Written informed consent was taken from the patient for the publication of this paper.

\section{Conflict of Interests}

The authors declare that they have no potential conflict of interests.

\section{References}

[1] A. Jemal, T. Murray, E. Ward et al., "Cancer statistics, 2005," CA: A Cancer Journal for Clinicians, vol. 55, no. 1, pp. 10-30, 2005.

[2] IARC, "Cancer Incidence, Prevalence and Mortality Worldwide (2002 estimates)," 2006, http://globocan.iarc.fr/.

[3] H. S. Al-Eid and S. O. Arteh, Cancer Incidence Report Saudi Arabia 2003, Kingdom of Saudi Arabia Ministry of Health National Cancer Registry, Riyadh, Saudi Arabia, 2003.

[4] Z. Zhu, M. Gandhi, M. N. Nikiforova, A. H. Fischer, and Y. E. Nikiforov, "Molecular profile and clinical-pathologic features of the follicular variant of papillary thyroid carcinoma: an unusually high prevalence of ras mutations," The American Journal of Clinical Pathology, vol. 120, no. 1, pp. 71-77, 2003.

[5] H. Sugimura, S. Tamura, T. Kodama, Y. Kakitsubata, K. Asada, and K. Watanabe, "Metastatic pancreas cancer from the thyroid. Clinical imaging mimicking non functioning islet cell tumor," Radiation Medicine, vol. 9, no. 5, pp. 167-169, 1991.

[6] R. Jobran, Z. W. Baloch, V. Aviles, E. F. Rosato, S. Schwartz, and V. A. LiVolsi, "Tall cell papillary carcinoma of the thyroid: metastatic to the pancreas," Thyroid, vol. 10, no. 2, pp. 185-187, 2000 . 
[7] A. Angeles-Angeles, F. Chable-Montero, B. Martinez-Benitez, and J. Albores-Saavedra, "Unusual metastases of papillary thyroid carcinoma: report of 2 cases," Annals of Diagnostic Pathology, vol. 13, no. 3, pp. 189-196, 2009.

[8] T. Borschitz, W. Eichhorn, C. Fottner et al., "Diagnosis and treatment of pancreatic metastases of a papillary thyroid carcinoma," Thyroid, vol. 20, no. 1, pp. 93-98, 2010.

[9] L. Chen and J. A. Brainard, "Pancreatic metastasis from papillary thyroid carcinoma diagnosed by endoscopic ultrasoundguided fine needle aspiration: a case report," Acta Cytologica, vol. 54, no. 4, pp. 640-644, 2010.

[10] A. S. Alzahrani, A. AlQaraawi, F. Al Sohaibani, H. Almanea, and $\mathrm{H}$. Abalkhail, "Pancreatic metastasis arising from a BRAF (V600E)-positive papillary thyroid cancer: the role of endoscopic ultrasound-guided biopsy and response to sorafenib therapy," Thyroid, vol. 22, no. 5, pp. 536-541, 2012.

[11] L. J. Layfield, S. L. Hirschowitz, and D. G. Adler, "Metastatic disease to the pancreas documented by endoscopic ultrasound guided fine-needle aspiration: a seven-year experience," Diagnostic Cytopathology, vol. 40, no. 3, pp. 228-233, 2012.

[12] A. A. Siddiqui, L. Olansky, R. N. Sawh, and W. M. Tierney, "Pancreatic metastasis of tall cell variant of papillary thyroid carcinoma: diagnosis by endoscopic ultrasound-guided fine needle aspiration," Journal of the Pancreas, vol. 7, no. 4, pp. 417422, 2006.

[13] H. Palmedo, J. Bucerius, A. Joe et al., "Integrated PET/CT in differentiated thyroid cancer: diagnostic accuracy and impact on patient management," Journal of Nuclear Medicine, vol. 47, no. 4, pp. 616-624, 2006.

[14] E. T. Kimura, M. N. Nikiforova, Z. Zhu, J. A. Knauf, Y. E. Nikiforov, and J. A. Fagin, "High prevalence of BRAF mutations in thyroid cancer: genetic evidence for constitutive activation of the RET/PTC-RAS-BRAF signaling pathway in papillary thyroid carcinoma," Cancer Research, vol. 63, no. 7, pp. 14541457, 2003. 


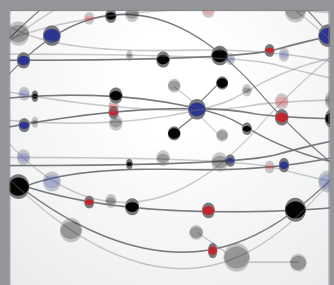

The Scientific World Journal
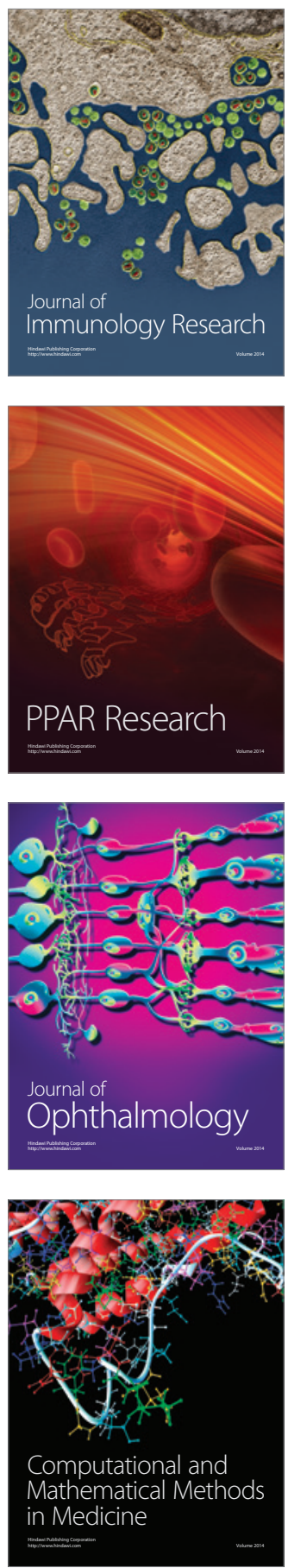

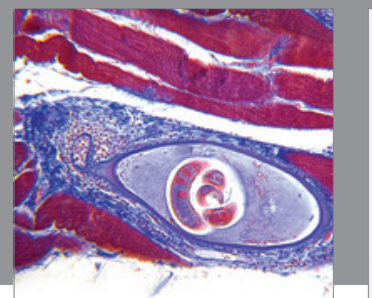

Gastroenterology

Research and Practice
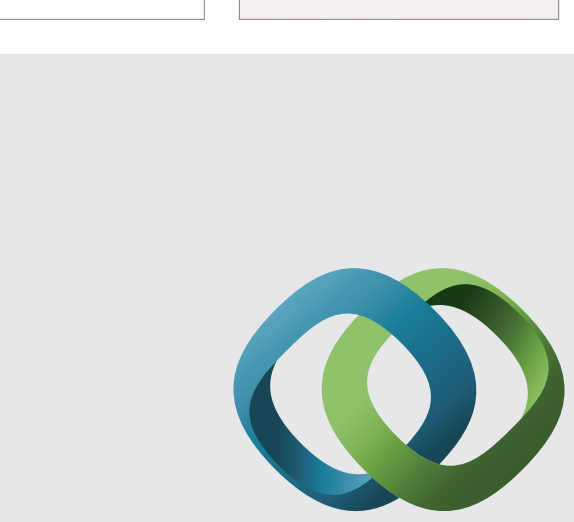

\section{Hindawi}

Submit your manuscripts at

http://www.hindawi.com
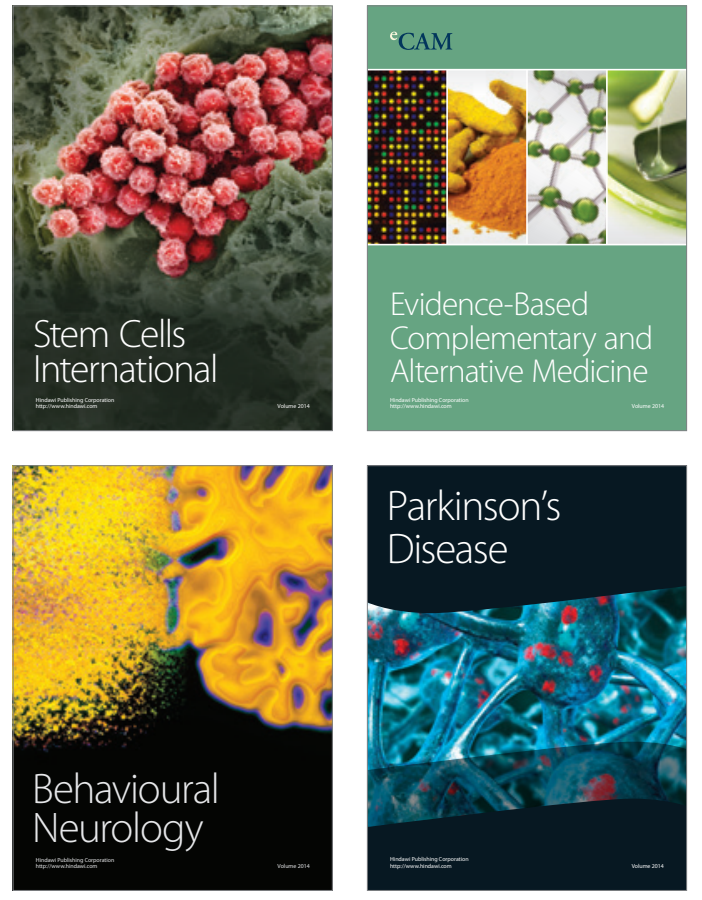
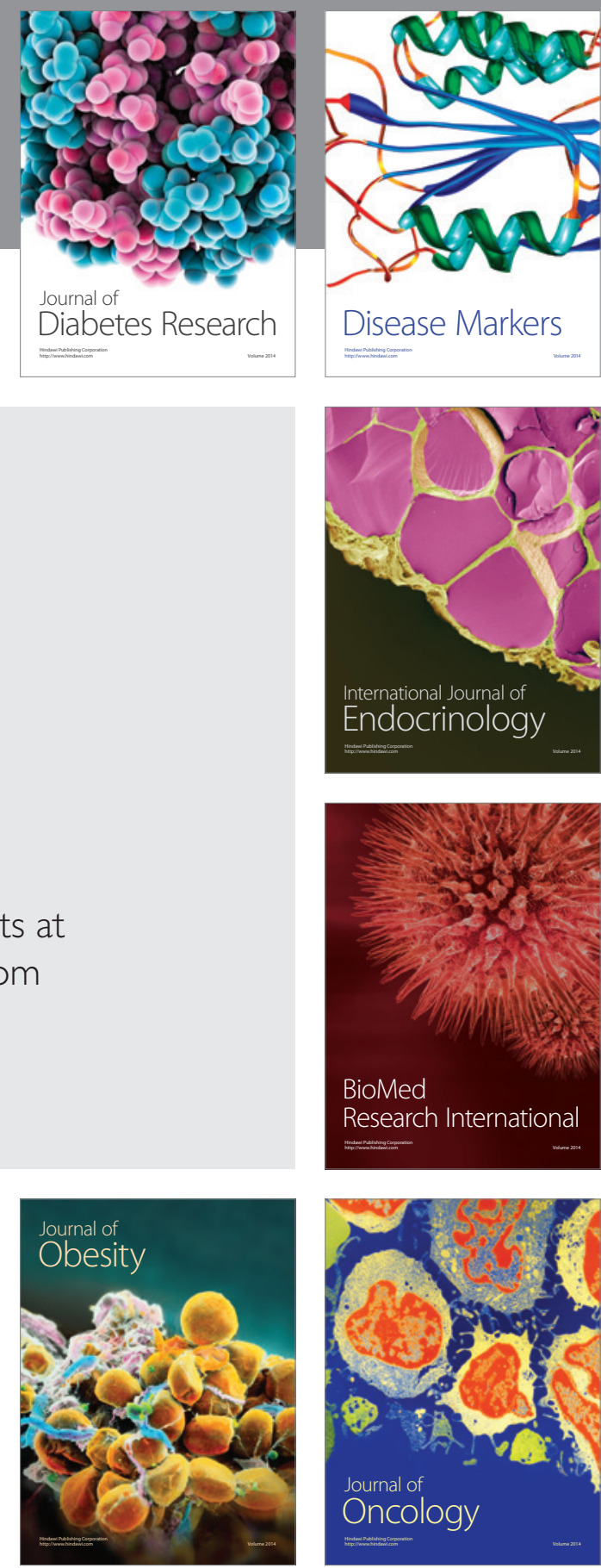

Disease Markers
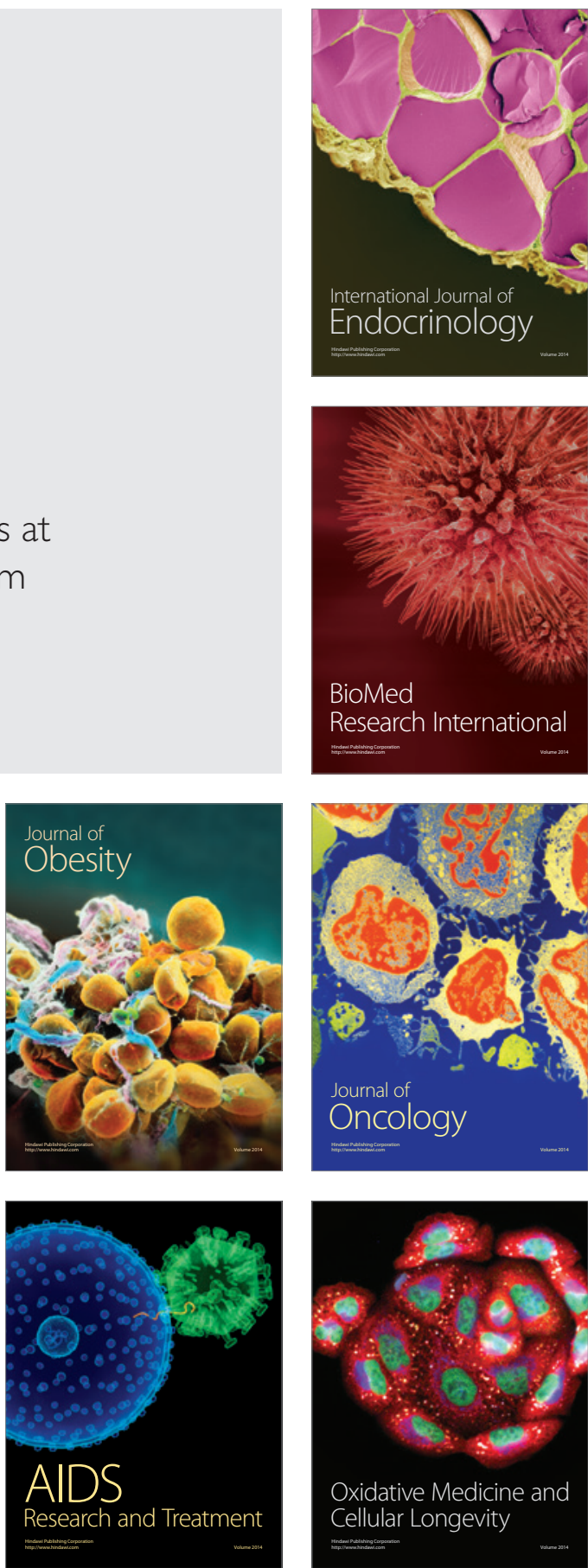